\title{
Analysis of non-isothermal mold filling process in Resin Transfer Molding (RTM) and Structural Reaction Injection Molding (SRIM)
}

\author{
W. Chang, N. Kikuchi
}

Abstract In this paper, we present a modeling and numerical simulation of a mold filling process in resin transfer molding/ structural reaction injection molding utilizing the homogenization method. Conventionally, most of the mold filling analyses have been based on a macroscopic flow model utilizing Darcy's law. While Darcy's law is successful in describing the averaged flow field within the mold cavity packed with a porous fiber preform, it requires experiments to obtain the permeability tensor and is limited to the case of porous fiber preform - it can not be used to model the resin flow through a double porous fiber preform. In the current approach, the actual flow field is considered, to which the homogenization method is applied to obtain the averaged flow model. The advantages of the current approach are: parameters such as the permeability and effective heat conductivity of the impregnated fiber preform can be calculated; the actual flow field as well as averaged flow field can be obtained; and the resin flow through a double porous fiber preform can be modelled. In the presentation, we first derive the averaged flow model for the resin flow through a porous fiber preform and compare it with that of other methods. Next, we extend the result to the case of double porous fiber preform. An averaged flow model for the resin flow through a double porous fiber preform is derived, and a simulation program is developed which is capable of predicting the flow pattern and temperature distribution in the mold filling process. Finally, an example of a three dimensional part is provided.

\section{1}

\section{Introduction}

Resin Transfer Molding (RTM) and Structural Reaction Injection Molding (SRIM) molding are relatively new process for manufacturing continuous fiber reinforced materials. They are among the several processes that have been developed for mass production of these materials. In RTM/SRIM, the mold is packed with dry fiber preforms which act as reinforcements. These preforms are impregnated progressively by the resin injected through injection ports of the mold. After the mold is

Communicated by S. N. Atluri, 20 October 1994

W. Chang, N. Kikuchi

Dept. of Mechanical Engineering and Applied Mechanics, The

University of Michigan, Ann Arbor, Michigan 48109-2125

Correspondence to: $\mathrm{W}$. Chang

The authors are supported by NSF (MSS-9021683) during this research. This support is gratefully acknowledged. completely filled, the resin solidifies in the curing phase and the product is taken out of the mold.

The analysis of a mold filling process is a very important step in the product development stages of RTM/SRIM products. It can provide useful information to a mold designer such as pressure distributions and flow front profiles within the mold cavity which can be used to predict operating parameters, possible location of defects, hard to fill regions, and proper locations of air tabs and injection ports. Conventionally, these data were obtained based on experience and repeated experiments which are time consuming and inefficient. In order to optimize the manufacturing process and reduce the cost, computer simulation of the mold filling process is necessary. So far, many computer programs have been developed which can simulate the mold filling process. However, most of these programs require as an input the permeability tensor which must be obtained by experiments.

In this paper, we develop a computational model to calculate the permeability effective heat conductivity of the impregnated fiber preform and use it to simulate a non-isothermal three dimensional mold flow problem. The ability to calculate the permeability and the effective heat conductivity can be very valuable to the mold designer, since it enables him to try all possible different fiber arrangements - or different micro structures - as well as different air tab and injection port arrangements to avoid defects. The mold designer may then conduct few experiments with some of the best fiber arrangements found in the calculation for more accurate analysis.

Here, we also consider the mold flow through double porous fiber preforms. In many mold filling processes, the fiber preforms used are woven structures made of fiber bundles which consist of much thinner fibers. For such fiber preforms, there are two different level of pore structures: the pore structure between the fiber bundles, and the pore structure inside the fiber bundles. In the mold filling, the pores between the fiber bundles are filled first and then the pores within the fiber bundles are filled up. This will create along the flow front an intermediate region where the fiber bundles are partially impregnated. Depending on the width of the region and fiber orientations, the air inside the fiber bundle can be trapped and cause defects in the product. In the current analysis, this partially impregnated region can be estimated, which, with some experimental data, can be used to predict the partial impregnation inside the fiber bundles.

Many researchers have investigated the mold filling process in the context of RTM and SRIM. Gonzalez, Castro, and Macosco (1985) studied the process using a disk-shaped mold. They de-coupled the chemical reactions and heat transfer from the 
mold flow and solved a 1-D mold filling problem using both analytical and numerical methods. Coulter and Güçeri (1988) studied the effects of anisotropic reinforcements on the mold flow. They considered an isothermal flow and developed a 2-D finite difference code to simulate the mold flow utilizing boundary fitted curvilinear coordinate systems. Young, Han, Fong, and Lee (1991) investigated the effects of permeability variations in a similar $2-\mathrm{D}$ setting. They simulated the mold flow utilizing the Fluid Analysis Network (FAN) method. Bruschke and Avani (1993) considered a non-isothermal flow and solved a 2-D momentum equation with a 3-D energy equation. They simulated the flow using the finite element/control volume method.

While many methods have been proposed and developed to simulate the mold filling process in these investigations, most of the analyses are based on the same macroscopic flow model - Darcy's law. Darcy's law states that the averaged resin velocity, $\bar{V}$ is proportional to the pressure gradient:

$$
\bar{V}=-\frac{1}{\mu} \bar{k} \nabla P
$$

where $\bar{k}$ is the permeability tensor, $P$ is the resin pressure, and $\mu$ is the viscosity. While Darcy's law is successful in describing the overall (or averaged) behavior of the mold flow, it does not give any information on the actual flow field in the pore structures of reinforcing fiber mats. All the interactions between the resin and fiber mat are summarized and represented by a single permeability tensor $\bar{k}$, which must be obtained by experiments.

Here, we take the microscopic approach to model the nonisothermal mold flow. In the microscopic approach, the flow through the fiber preform is analyzed based on the boundary conditions which account for all the fibers in the flow field. It may seem beyond the limitations of the computational capability to calculate the actual flow field within the fiber mat while considering the reinforcing fibers as boundary conditions. However, there is a method to calculate the averaged flow field while taking care of the actual flow field. Using the homogenization method, one can simulate the mold filling process in terms of averaged flow field and calculate the actual flow field between the reinforcing fibers whenever necessary.

The homogenization theory appeared in the 1970's and has been the subject of considerable research in different areas of applied mechanics. The theory deals with partial differential equations of physics in heterogeneous materials with periodic structures when the characteristic length of the period is small. The fundamentals of the theory can be found in the works of Lions (1981) and some generalizations can be found in the works of Lene (1984). Application of the method to the flow and transport through porous media was first done by Keller (1980) and Tartar (1980) who showed the derivation of Darcy's law as a macroscopic equation from Stoke's equations as a micro-model. After the pioneering work of Keller and Tartar many applications followed (Arbogast (1989), Hornung (1991)).

In the homogenization method, it is assumed that the flow domain is locally formed by spatial repetition of a "microscopic cell" which is very small compared to the overall dimension of the flow domain. With this assumption, the governing equations for the actual flow field are converted to a set of 'microscopic equations' and 'macroscopic equations'. The microscopic equations govern the characteristic flow field within the microstructure, and the macroscopic equations govern the averaged flow field of the mold filling. From the solutions of the microscopic equations, the permeability and averaged heat conductivity of the flow domain are calculated which, in turn, are used to solve the averaged flow field in the macroscopic equations. After the simulation, the solutions of the microscopic equations can be post-processed to give the actual flow field within the microstructure. Advantages of the homogenization method are: parameters such as permeability of the flow domain and effective conductivity of the impregnated fiber preform can be calculated; the actual flow field within the pore structure can be obtained as well as the averaged flow field; and the method is based on a rigorous mathematical theory.

In the following sections, we utilize the homogenization method to obtain the governing equations of a non-isothermal mold flow through double porous fiber preforms. First, we give a brief derivation of the homogenized governing equations for the mold flow through porous fiber mat and compare them with those of other methods in terms of permeability and averaged heat conductivity. Next, we derive the governing equations for the mold flow through a double porous fiber mat. Then, a computer program is developed which is capable of predicting the flow pattern and the temperature distribution within the mold cavity. The program is based on the finite element/control volume method and the Crank Nicholson method and is capable of simulating the mold filling of three dimensional parts. Finally, an example is provided for a simplified composite crossmember of a passenger van. In the example, the flow pattern, temperature distribution, and the actual flow field within the mold cavity is obtained and plotted.

\section{2}

\section{Resin flow through porous fiber preform}

\section{1}

\section{Mathematical model}

In RTM/SRIM, the mold is pre-packed with fiber preforms. Throughout the mold filling process, the fiber preform is assumed to be rigid - it is not deformed or shifted by the resin flow. The flow of resin through the porous fiber mat is usually very slow and can be modeled as Stokes' flow which is an inertia-less viscous flow. The inertia effect is negligible because the Reynold's number of the resin flow is small, and the effect of the surface tension is negligible compared to the dominant viscous force. With these assumptions, the momentum balance equation and the continuity equation of the resin flow can be written as:

$$
\begin{aligned}
& -\nabla P+\mu \nabla \cdot(\nabla \bar{v})+\bar{f}=0 \\
& \nabla \cdot \bar{v}=0
\end{aligned}
$$

where $\bar{v}$ is the actual velocity, $P$ the pressure, $\mu$ the viscosity, and $\bar{f}$ the specific weight of the resin. The boundary conditions are: pressure is zero at the free flow front, and the normal component of velocity is zero at the mold walls. Either injection pressure or flow rates are prescribed at the injection ports depending on the port types. 
In general, the mold filling process is not an isothermal process. As the resin fills the mold, heat transfer takes place between the resin, fiber preform and mold walls. Heat may be generated by the resin curing which is an exothermic process. As a result, temperature variations are created throughout the mold cavity. These temperature variations influence the flow pattern by changing the viscosity which is a strong function of temperature.

In analyzing the heat transfer, it is assumed that the resin and fiber reach local thermodynamic equilibrium as soon as the fiber is impregnated. This is justified because the heat transfer coefficient between the resin and fiber is fairly large compared to the thickness of the fibers. With this assumption, the energy equation takes the following form:

$\rho c_{p} \frac{\partial T}{\partial t}+\rho c_{p}(\bar{v} \cdot \nabla T)=-\nabla \cdot(-\bar{\kappa} \nabla T)+\dot{s}$

where $T$ is the temperature and $t$ is the time. $\rho$ and $c_{p}$ are the density and heat capacity respectively. $\bar{\kappa}$ is the conductivity. The coefficients $\rho, c_{p}$ and $\bar{\kappa}$ are equal to $\rho_{r}, c_{p r}$ and $\bar{\kappa}_{r}$ at the resin and equal to $\rho_{f}, c_{p f}$ and $\bar{\kappa}_{f}$ at the fiber. $\rho_{r}, c_{p r}, \bar{\kappa}_{r}$ and $\rho_{f}$, $c_{p p} \bar{\kappa}_{f}$ are the density, heat capacity, and conductivity of the resin and of the fiber respectively. $\bar{v}$ is the velocity of the resin flow and $\dot{s}$ is the specific heat generated in the resin due to curing. Both $\bar{v}$ and $\dot{s}$ have zero value at the fiber. The first term of (4) represents the change in internal energy, and the second term represents the contribution due to the convection of the resin. On the right hand side, the first term represents the contribution due to conduction. The last term represents the energy generated due to curing.

To solve the energy Eq. (4), boundary conditions must be specified at the injection ports, the mold walls, and the flow front. At the injection ports and mold walls, temperature is specified. For a more complete analysis, the mold plates can be included in the heat transfer analysis instead of specifying mold wall temperatures. At the flow front, the heat transfer from the flow front to the ambient is neglected. However, the initial internal energy of the fiber preforms is taken into account during the impregnation at the flow front. This is done by considering a local heat balance which assigns a convection type boundary condition at the flow front.

The energy Eq. (4) is coupled with the momentum Eq. (2) in terms of viscosity which is a function of temperature. The viscosity can be obtained using an Arrhenius type relation which describes the viscosity, $\mu$, as an exponential function of temperature:

$\mu=\mu_{0} \exp (-a \theta)$

where $\theta=T-T_{0}$ with $T_{0}$ being the reference temperature at which the viscosity is $\mu_{0}$.

In addition to being a function of temperature, the viscosity of resin also depends on the degree of cure. However, we assume that the curing starts after the mold is completely filled and do not include curing effects in the current analysis. If this effect becomes significant, a chemorheological model should be introduced to Eq. (5). An analysis which take into account a chemorheological model can be found in Bruschke and Advany (1993).
The heat transfer needs to be analyzed in three dimensions, even for the thin shell type mold cavities. For thin mold cavities, the temperature gradient in the thickness direction is large, which makes the heat conduction from the mold wall to the resin as important as the convection in the in-plane flow.

\section{2}

\section{The homogenization method}

It is almost impossible to solve the governing Eqs. (2)-(5) due to the complexity of the flow domain which consists of the cavity between the reinforcing fibers. Here, we try to remove this difficulty by applying the homogenization method, which incorporates a special averaging technique.

In the homogenization method, it is assumed that the fiber mat is made of spatial repetitions of a unit cell, and that the scale length of the unit cell $(l)$ is much smaller than the overall dimension of the flow domain $(L)$. See Fig. 1 . In order to capture the details within the periodic micro structure, a stretched scale $(\bar{y})$ is utilized. The coordinate $\bar{y}$ is defined by:

$\bar{y}=\frac{\bar{x}}{\varepsilon} \quad$ where $\quad \varepsilon=\frac{l}{L}$

Using the stretched coordinate $\bar{y}$, the details of the unit cell are shown in Fig. 2. In the figure, $Y$ is the unit cell, $Y_{1}$ is the cavity between the reinforcing fibers which is to be filled by the resin, and $Y_{2}$ is the solid part consisting of the fiber. $\Gamma$ is the boundary between $Y_{1}$ and $Y_{2}$.

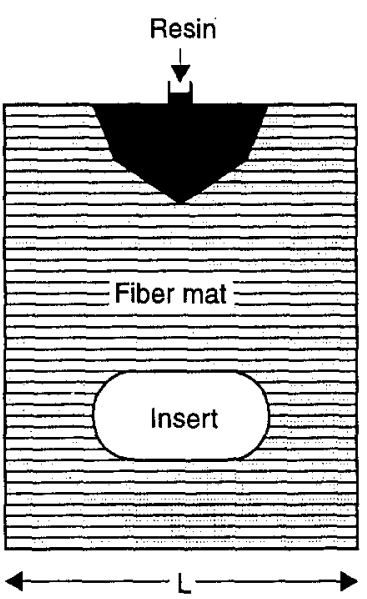

Configuration of a mold cavity

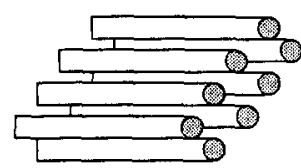

Details of the fiber mat

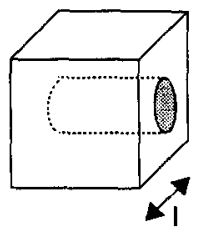

Unit cell of the fiber mat
Fig. 1. Periodic structure of a fiber mat

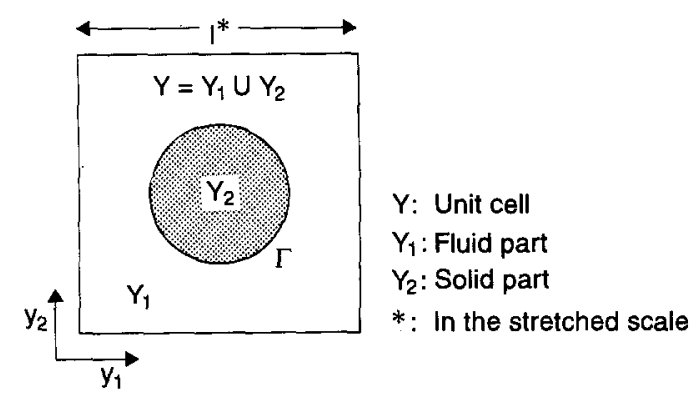

Fig. 2. Cross section of a unit cell 
Since the flow domain has a periodic micro structure, the pressure, velocity and temperature distribution tend have two dependencies on space variables: the dependency in the macroscopic level $(\bar{x})$, and the dependency in the microscopic level $(\bar{y}, \bar{y}=\bar{x} / \varepsilon)$. Furthermore, the dependency on $\bar{y}$ is $Y$ periodic. In order to represent the dependency in the microscopic level, symbols $P^{\varepsilon}(\bar{x}), \bar{v}^{\varepsilon}(\bar{x})$ and $T^{\varepsilon}(\bar{x})$ are used instead of $P(\bar{x}), \bar{v}(\bar{x})$ and $T(\bar{x})$.

The objective of the homogenization method is to remove the dependency on $\bar{y}$ by introducing an averaging technique over the unit cell. Heuristically, the method is based on the consideration of two length scales associated with microscopic and macroscopic phenomena as $\varepsilon$ approaches zero. In the following sections, the method is applied to the momentum and continuity Eqs. (2) - (3) and to the energy Eq. (4).

\subsection{1}

\section{The momentum equation and continuity equation}

The first step of the homogenization method is to expand the pressure, $P^{\varepsilon}(\bar{x})$ and the velocity, $\bar{v}^{\varepsilon}(\bar{x})$ into a formal power series of $\varepsilon$ (here, the argument for time, $t$ is dropped for the simplicity of notation).

$$
\begin{aligned}
& P^{\varepsilon}(\bar{x})=P^{(0)}(\bar{x})+\varepsilon P^{(1)}(\bar{x}, \bar{y})+\varepsilon^{2} P^{(2)}(\bar{x}, \bar{y})+\cdots \\
& \bar{v}^{\varepsilon}(\bar{x})=\varepsilon^{2} \bar{v}^{(0)}(\bar{x}, \bar{y})+\varepsilon^{3} \bar{v}^{(1)}(\bar{x}, \bar{y})+\varepsilon^{4} \bar{v}^{(2)}(\bar{x}, \bar{y})+\cdots
\end{aligned}
$$

Here, $P^{(i)}$ and $\vec{v}^{(i)}$ are periodic functions with respect to argument $\bar{y}$. Since $\bar{y}$ is defined as $\bar{y}=\bar{x} / \varepsilon$, the differential operator in Eqs. (2)-(3) becomes:

$\frac{\partial \phi^{\varepsilon}(\bar{x})}{\partial x_{i}}=\frac{\partial \phi^{\varepsilon}(\bar{x}, \bar{y})}{\partial x_{i}}+\frac{1}{\varepsilon} \frac{\partial \phi^{\varepsilon}(\bar{x}, \bar{y})}{\partial y_{i}}$

where $\phi^{\varepsilon}(\bar{x})$ is an arbitrary function of $\bar{x}$ and $\bar{y}$. Introducing (7)-(9) to Eqs. (2) - (3) and collecting terms of the same order of $\varepsilon$ yields:

$-\frac{\partial P^{(1)}}{\partial y_{i}}+\frac{\partial}{\partial y_{j}}\left(\mu \frac{\partial v_{i}^{(0)}}{\partial y_{j}}\right)+\left(f_{i}-\frac{\partial P^{(0)}}{\partial x_{i}}\right)=0$

$\frac{\partial v_{i}^{(0)}}{\partial y_{i}}=0$

$\left.\bar{v}^{(0)}\right|_{\Gamma}=0$

$\frac{\partial v_{i}^{(0)}}{\partial x_{i}}+\frac{\partial v_{i}^{(1)}}{\partial y_{i}}=0$

$\left.\bar{v}^{(1)}\right|_{\Gamma}=0$

The first three Eqs. (10)-(12) can be written in a variational form which is:

$$
\begin{aligned}
v_{i}^{(0)} \in V_{y}: \quad & -\int_{\Omega} \frac{\partial P^{(1)}}{\partial y_{i}} w_{i} d \Omega+\int_{\Omega} \frac{\partial}{\partial y_{j}}\left(\mu \frac{\partial v_{i}^{(0)}}{\partial y_{j}}\right) w_{i} d \Omega \\
& +\int_{\Omega}\left(f_{i}-\frac{\partial P^{(0)}}{\partial x_{i}}\right) w_{i} d \Omega=0, \quad \forall w_{i} \in V_{y}
\end{aligned}
$$

where:

$V_{y}=\left\{w_{i} \mid w_{i} \in\left(H^{1}\left(Y_{1}\right)\right)^{3}, Y\right.$-periodic, $w_{i}=0$ on $\left.\Gamma, \frac{\partial w_{i}}{\partial y_{i}}=0\right\}$

To remove the $\bar{y}$-dependencies in Eq. (15), a "mean" or "average" operator is introduced. The mean operator, " " is defined as:

$\tilde{\phi}=\frac{1}{|Y|} \int_{Y} \phi d Y$

where $|Y|$ is the measure of $Y$, and $\phi$ is any $Y$-periodic function. Note that after applying the mean operator, $\tilde{\phi}$ is not a function of $\bar{y}$ any more. Applying the mean operator and the divergence theorem to Eq. (15) gives:

$\left(f_{i}-\frac{\partial P^{(0)}}{\partial x_{i}}\right) \int_{Y} w_{i} d Y-\int_{Y} \frac{\partial w_{i}}{\partial y_{j}} \mu \frac{\partial v_{i}^{(0)}}{\partial y_{j}} d Y=0$

Suppose that $\chi_{i}^{m}$ is the solution of:

$\chi_{i}^{m} \in V_{y}: \quad \int_{Y_{1}} \frac{\partial \chi_{i}^{m}(\bar{y})}{\partial y_{j}} \frac{\partial w_{i}(\bar{y})}{\partial y_{j}} d Y=\int_{Y_{1}} w_{m}(\bar{y}) d Y \quad \forall w_{i} \in V_{y}$

Then, due to the linearity of Eq. (18), $v_{i}^{0}$ can be written as:

$v_{i}^{(0)}=\frac{\chi_{i}^{m}}{\mu}\left(f_{m}-\frac{\partial P^{(0)}}{\partial x_{m}}\right)$

Applying the mean operator to Eq. (20) yields:

$v_{i}^{D}=\frac{K_{i m}}{\mu}\left(f_{m}-\frac{\partial P}{\partial x_{m}}\right)$

where:

$v_{i}^{D}=\varepsilon^{2} \tilde{v}_{i}^{(0)}$

$K_{i m}=\varepsilon^{2} \tilde{\chi}_{i}^{m}$

$P=\tilde{P}^{(0)}$

Note that Eq. (21) is identical to Darcy's law. The averaged velocity $\vec{v}^{D}$ defined in (22) is called the "Darcean velocity".

From the remaining two Eqs. (13)-(14), the continuity equation for the Darcean velocity field can be derived. Applying the mean operator and the divergence theorem to Eqs. (13)-(14) yields:

$\frac{\partial v_{i}^{D}}{\partial x_{i}}=0$

The continuity Eq. (25) can be combined with Eq. (21) to give the pressure formulation:

$\frac{\partial}{\partial x_{i}}\left[\frac{K_{i m}}{\mu}\left(f_{m}-\frac{\partial P}{\partial x_{m}}\right)\right]=0$ 
This pressure formulation is easier to solve and thus is used in the simulation because it deals with scalar quantity $(P)$ rather than vector quantity such as $\left(\bar{v}^{D}\right)$.

As a result of applying the homogenization method to the momentum equation and to the continuity equation, Darcy's law (21) and the continuity equation for the Darcean velocity field (25) is derived. The permeability tensor of Darcy's equation is calculated in (23) using the solution of Eq. (19) which is called the "microscopic equation". The permeability tensor, in turn, is used to calculate the pressure distribution in Eq. (26) which is called the "macroscopic equation". After the pressure distribution is calculated, the Darcean velocity field and the actual velocity field can be obtained by Eqs. (21) and (20) respectively.

In order to verify the current formulation, permeability tensors are calculated and compared to the results of Sahraoui and Kaviany (1991). They calculated permeability tensors by solving Navier-Stokes' equation for flow over various arrangements of cylinders using the finite difference approximation . In Fig. 4, normalized permeability $\left(K / \mathbb{R}^{2}\right)$ of the current method is compared to the result of Sahraoui and Kaviany. The plot is obtained by calculating the transverse permeability $(K)$ and normalizing it with respect to the size of the unit cell $(l)$. See Fig. 3. The flow domains used in the calculations consist of in-line array cylinders with various volume fractions. Even though the current flow model does not take into account any inertia effects which are considered by Sahraoui and Kaviany, the plot shows that the two results agree within $10 \%$, which justifies the assumption made to model the resin flow as an inertia-less viscous flow.

The permeabilities calculated here, like most of the computed permeabilities, gives rough approximations, because the formulation relies on heavy assumptions (rigid and periodic fiber structure, etc.). However, in many cases, they can still provide reasonable trends to the change of micro structure, and can be used as a guideline in the initial mold design stage when the mold designer is checking all the possible fiber arrangements. This will reduce the number of experiments while providing more design options to the mold designer.

\subsection{2}

\section{The energy equation}

As with the momentum and continuity equation, the temperature variable of the energy Eq. (4) is expanded into a formal power series of $\varepsilon$ :

$T^{\varepsilon}(\bar{x})=T^{(0)}(\bar{x})+\varepsilon T^{(1)}(\bar{x}, \bar{y})+\varepsilon^{2} T^{(2)}(\bar{x}, \bar{y})+\cdots$

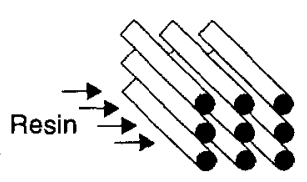

In-line array of cylinders

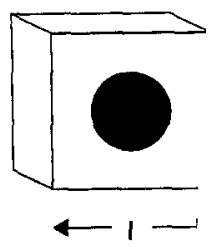

Unit cell
Fig. 3. In-line array of cylinders

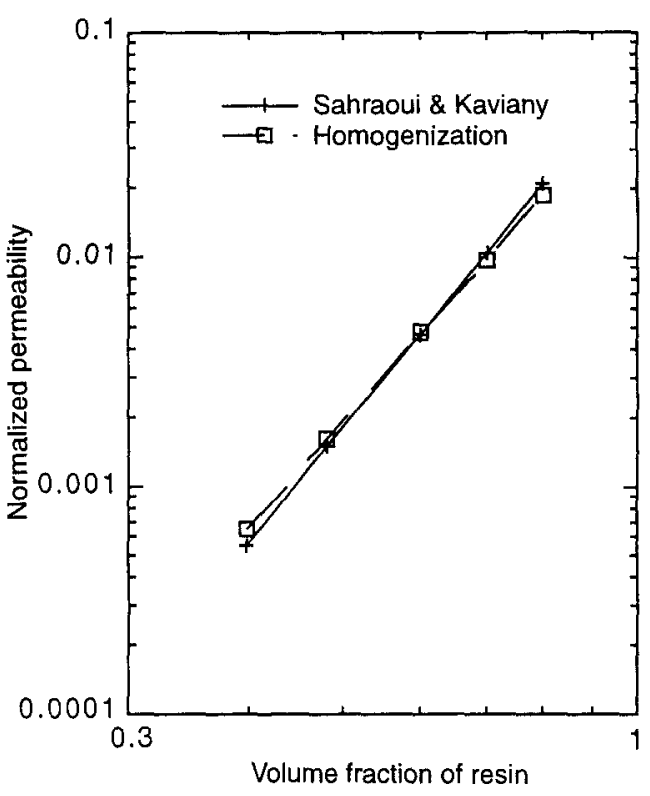

Fig. 4. Transverse permeability for in-line array of cylinders

Introducing (9) and (27) to Eq. (4) and collecting terms of the same order of $\varepsilon$ yields:

$$
\begin{aligned}
& \frac{\partial}{\partial y_{i}}\left[\kappa_{i j}\left(\frac{\partial T^{(0)}}{\partial x_{j}}+\frac{\partial T^{(1)}}{\partial y_{j}}\right)\right]=0 \\
& \rho c_{p} \frac{\partial T^{(0)}}{\partial t}+\rho c_{p}\left[v_{i}\left(\frac{\partial T^{(0)}}{\partial x_{i}}+\frac{\partial T^{(1)}}{\partial y_{i}}\right)\right] \\
& \quad=-\frac{\partial}{\partial x_{i}}\left[-\kappa_{i j}\left(\frac{\partial T^{(0)}}{\partial x_{j}}+\frac{\partial T^{(1)}}{\partial y_{j}}\right)\right]+\dot{s}
\end{aligned}
$$

The first Eq. (28) can be written in a weak form:

$\int_{\Omega} \frac{\partial}{\partial y_{i}}\left[\kappa_{i j}\left(\frac{\partial T^{(0)}}{\partial x_{j}}+\frac{\partial T^{(1)}}{\partial y_{j}}\right)\right] \tau d \Omega=0, \quad \forall \tau \in W_{y}$

where

$W_{y}=\left\{w \mid w \in\left(H^{1}(Y)\right)^{3}, Y\right.$-periodic $\}$

Applying the mean operator and the divergence theorem to Eq. (30) yields:

$\int_{Y} \frac{\partial \tau}{\partial y_{i}} \kappa_{i j} \frac{\partial T^{(1)}}{\partial y_{j}} d Y=-\int_{Y} \frac{\partial \tau}{\partial y_{i}} \kappa_{i j} \frac{\partial T^{(0)}}{\partial x_{j}} d Y$

Suppose that $\psi^{p}$ is the solution of:

$\int_{Y} \frac{\partial \tau}{\partial y_{i}} \kappa_{i j} \frac{\partial \psi^{p}}{\partial y_{j}} d Y=-\int_{Y} \frac{\partial \tau}{\partial y_{i}} \kappa_{i p} d Y$

Then, due to the linearity of Eq. (32), $T^{(1)}$ can be given as:

$T^{(1)}=\psi^{p} \frac{\partial T^{(0)}}{\partial x_{p^{\prime}}}+f(\bar{x})$

where $f(\bar{x})$ is an arbitrary function of $\bar{x}$. 
The second Eq. (29) can be written in a weak form:

$$
\begin{aligned}
& \int_{\Omega} \rho c_{p} \frac{\partial T^{(0)}}{\partial t} \tau d \Omega+\int_{\Omega} \rho c_{p}\left[v_{i}\left(\frac{\partial T^{(0)}}{\partial x_{i}}+\frac{\partial T^{(1)}}{\partial y_{i}}\right)\right] \tau d \Omega \\
& \quad+\int_{\Omega} \frac{\partial \tau}{\partial x_{i}} \kappa_{i j}\left(\frac{\partial T^{(0)}}{\partial x_{j}}+\frac{\partial T^{(1)}}{\partial y_{j}}\right) d \Omega \\
& =\int_{\Omega} \dot{s} \tau d \Omega+\int_{\partial \Omega} \kappa_{i j}\left(\frac{\partial T^{(0)}}{\partial x_{j}}+\frac{\partial T^{(1)}}{\partial y_{j}}\right) n_{i} \tau d \partial \Omega, \quad \forall \tau \in W_{x}
\end{aligned}
$$

where

$$
W_{x}=\left\{w \mid w \in\left(H^{1}(\Omega)\right)^{3}, w=0 \text { at mold walls }\right\}
$$

$\partial \Omega$ denotes the boundary of the flow domain $\Omega$. The last term of Eq. (35) represents the boundary condition. At the mold walls, the term becomes zero because the temperature is prescribed. At the flow front, the term can be replaced by a convection type boundary condition which is obtained by considering the local balance of energy.

After combining Eqs. (34) and (35), the mean operator and the divergence theorem are applied to give:

$$
\begin{aligned}
& \int_{\Omega}\left[\frac{1}{|Y|} \int_{Y} \rho c_{p} d Y\right] \frac{\partial T^{(0)}}{\partial t} \tau d \Omega+\int_{\Omega}\left[\frac{1}{|Y|} \int_{Y} \rho c_{p} v_{i} d Y\right] \frac{\partial T^{(0)}}{\partial x_{i}} \tau d \Omega \\
& \quad+\int_{\Omega}\left[\frac{1}{|Y|} \int_{Y}\left(\kappa_{i p}+\kappa_{i j} \frac{\partial \psi^{p}}{\partial y_{j}}\right) d Y\right] \frac{\partial T^{(0)}}{\partial x_{p}} \frac{\partial \tau}{\partial x_{i}} d \Omega \\
& =\int_{\Omega}\left[\frac{1}{|Y|} \int_{Y} \dot{s} d Y\right] \tau d \Omega-\int_{\partial \Omega_{f}} c_{p f} \rho_{f} \phi_{f} v_{i} n_{i}\left(T^{(0)}-T_{g}\right) d \partial \Omega
\end{aligned}
$$

where $\partial \Omega_{f}$ denotes the flow front boundary, $\phi_{f}$ denotes the volume fraction of the fiber part, and $T_{g}$ denotes the initial temperature of the fiber. In Eq. (37), the last term of (35) is replaced by the convection type boundary condition which takes into account the initial temperature of the fibers. Equation (37) can be rewritten as:

$$
\begin{gathered}
\int_{\Omega}\left(\rho c_{p}\right)^{H} \frac{\partial T}{\partial t} \tau d \Omega+\int_{\Omega} \rho_{r} c_{p r} v_{i}^{D} \frac{\partial T}{\partial x_{i}} \tau d \Omega+\int_{\Omega} \kappa_{i p}^{H} \frac{\partial T}{\partial x_{p}} \frac{\partial \tau}{\partial x_{i}} d \Omega \\
=\int_{\Omega} \dot{s}^{H} \tau d \Omega-\int_{\partial \Omega_{f}} \frac{c_{p f} \rho_{f} \phi_{f}}{\phi_{r}} v_{i}^{D} n_{i}\left(T-T_{g}\right) d \partial \Omega
\end{gathered}
$$

where:

$$
\left(\rho c_{p}\right)^{H}=\frac{1}{|Y|} \int_{Y} \rho c_{p} d Y
$$

$\dot{s}^{H}=\frac{1}{|Y|} \int_{Y} \dot{s} d Y$

$$
\kappa_{i p}^{H}=\frac{1}{|Y|_{Y}} \int_{Y}\left(\kappa_{i p}+\kappa_{i j} \frac{\partial \psi^{p}}{\partial y_{j}}\right) d Y
$$

$\phi_{\gamma}$ is the voume fraction of the resin part, and $T$ is identical to $T^{(0)}$. Note that the boundary conditions of (38) need not be periodic since $T^{(0)}$ is a function of $\bar{x}$ only.
As a result of applying the homogenization method to the energy equation, another set consisting of a macroscopic equation and a microscopic equation is obtained. Equation (38) is the macroscopic equation which describes the averaged (or overall) heat transfer within the mold cavity. In (38), homogenized constants defined in (39)-(41) are used to represent Eq. (37). $\left(\rho c_{p}\right)^{H}$ is the homogenized version of $\left(\rho c_{p}\right)$ and is equal to the volume average of $\left(\rho c_{p}\right)$ between the resin and fiber. $\dot{s}^{H}$ is the homogenized version of $\dot{s}$ and is equal to $\left(\dot{s} \phi_{\tau}\right) . \kappa_{i j}^{H}$ is called the "homogenized conductivity" and represents the effective conductivity of the impregnated fiber preform. Note that $\kappa_{i j}^{H}$ is not a simple volume average of $\kappa_{i j}$, It is calculated from the solution of the microscopic Eq. (33) which governs the characteristic heat conduction within the unit cell.

In order to verify the current method, the current formulation (33), (38)-(41) is compared to that of other method. Since the homogenized coefficients $(\rho c)^{H}$ and $\dot{s}^{H}$ which are obtained by simple volume averaging are widely accepted among researchers, comparison is made in terms of the homogenized (or effective) conductivity. In Fig. 5, effective conductivity is calculated and plotted using the homogenization method and the self-consistent model method, which is based on a simplification of a medium consisting of cylinders. The plot is obtained considering the transverse conduction in the in-line array of cylinders with various volume fractions. The conductivity was $1.0(\mathrm{~W} / \mathrm{mK})$ for the fiber part and $0.2(\mathrm{~W} / \mathrm{mK})$ for the resin part. In the plot, the two results show good agreement, within $4 \%$.

\section{3}

\section{Resin flow through double porous fiber preform}

In this section, we extend the results of the previous sections to the mold flow through double porous fiber preforms. A double porous fiber preform is a fiber preform whose reinforcing fiber is actually a fiber bundle - which is a porous media itself - made of much thinner fibers. See Fig. 6. Due to

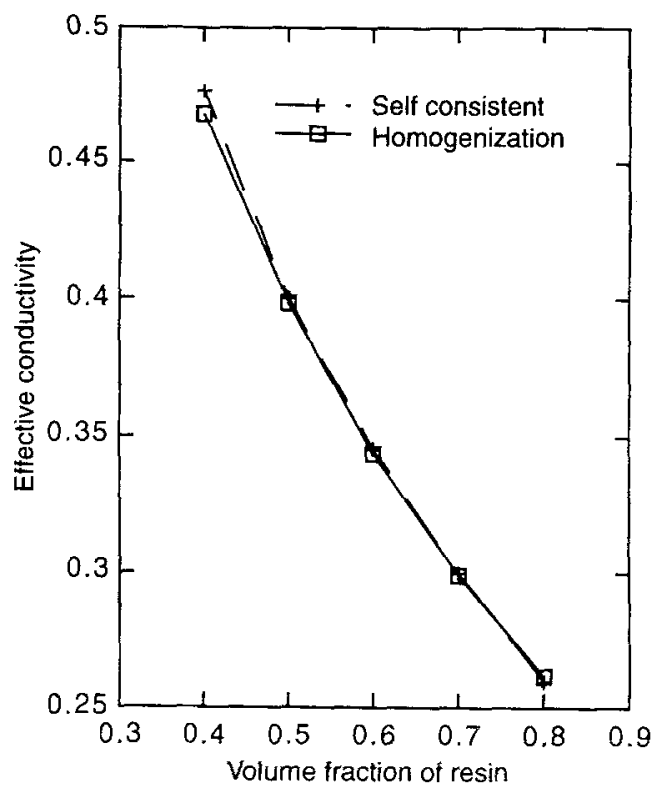

Fig. 5. Normalized conductivity for in-line array of cylinders 
this added complexity, several difficulties arise in the analysis. Now, the flow domain has become even more complicated. It consists of pore structures in two regimes: one between the reinforcing fiber bundles, and the other within the reinforcing fiber bundles. To simulate the mold filling process, one has to consider both regimes of flow which are interacting with each other. To overcome this difficulty, we apply the homogenization method twice to derive a set of governing equations for the averaged flow field.

As a flow domain, a double porous fiber preform has micro structures in two different scales, namely, the "first level micro structure" and the "second level micro structure". The first level micro structure is the pore structure between the reinforcing fiber bundles whereas the second level micro structure is the pore structure within the fiber bundles. In both levels of micro structure, it is assumed that the micro structures are made of repetitions of a unit cell, which implies that they have periodic structures. It is also assumed that the scale length $(l)$ of the unit cell of the first level micro structure is much smaller than the macroscopic scale length $(L)$, and that the scale length $\left(l^{\prime}\right)$ of the unit cell of the second level micro structure is much smaller than $l$.

In order to capture the details within both levels of micro structure, two stretched scales, $\bar{y}$ and $\bar{z}$ are employed. The coordinate $\bar{y}$ is used for the first level micro structure whereas the coordinate $\bar{z}$ is used for the second level micro structure. These coordinates are defined by:

$\bar{y}=\frac{\bar{x}}{\varepsilon} \quad$ where $\quad \varepsilon=\frac{l}{L}$

$\bar{z}=\frac{\bar{y}}{\delta} \quad$ where $\quad \delta=\frac{l^{\prime}}{l}$

In Fig. 7, the unit cells of both levels of micro structures are shown in scaled coordinates. The unit cell of the first level microstructure $Y$ consists of fluid part $Y_{1}$ and fiber bundle part $Y_{2}$ with boundary $\Gamma$. The unit cell of the second level micro structure $Z$ consists of fluid part $Z_{1}$ and fiber (or solid) part $Z_{2}$ with boundary $\Sigma$.

While the flow domain is much more complicated for the double porous fiber mat, the governing equations for the resin flow remain the same. The governing equations are:

$$
-\nabla P+\mu \nabla \cdot(\nabla \bar{v})+\bar{f}=0
$$

$\nabla \cdot \bar{v}=0$

$\rho c_{p} \frac{\partial T}{\partial t}+\rho c_{p}(\bar{v} \cdot \nabla T)=-\nabla \cdot(-\bar{\kappa} \nabla T)+\dot{s}$

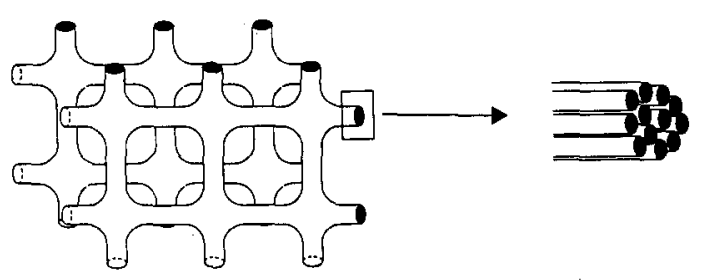

Details of a fiber mat

Details of the fiber bundle

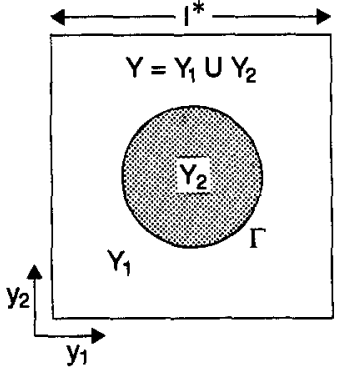

Cross section of a unit cell of the first level micro structure

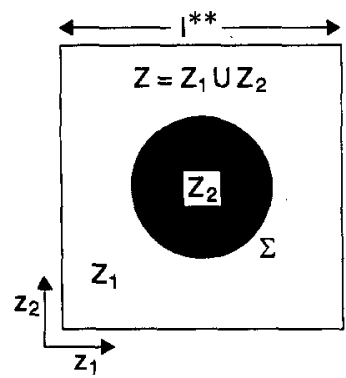

Cross section of a unit cell of the second level micro structure

\section{*: Length in y scale **: Length in z scale}

Fig. 7. Unit cells of the first and second level micro structures

\section{1}

\section{The momentum and continuity equation}

The homogenization method will be applied to the momentum and continuity Equations (44)-(45) noting that there are two different regimes of flow domain. First, the homogenization method is applied in the second level micro structure, which describe the flow within the fiber bundle. As a result, the macroscopic and microscopic equations are obtained. The macroscopic equation describes the averaged flow within the fiber bundle, whose permeability is calculated from the solution of the corresponding microscopic equation. Formal derivation is identical to that of sect. 2.2.1 except for the scaling parameter $(\varepsilon)$ and thus is omitted. The obtained macroscopic equation is identical to Darcy's law:

$\frac{\partial v_{i}^{d}}{\partial x_{i}}=0 \quad$ where $\quad v_{j}^{d}=-(\varepsilon \delta)^{2} \frac{k_{i j}}{\mu} \frac{\partial P}{\partial x_{j}}$

where $v_{i}^{d}$ is the averaged velocity, and $k_{i j}$ is the calculated permeability inside the fiber bundle.

Remark: Depending on the fiber surface and resin type, the surface tension can have significant effect on the flow field inside the fiber bundle. The effect can be taken into account by assigning negative pressure at the resin/air boundary within the fiber bundle. This requires experiments to determine the magnitude of the pressure, and is not included in the example of sect. 5 .

Now we have two different types of flow within the mold cavity interacting with each other. One is the Darcy's flow within the fiber bundle, and the other is Stokes' flow between the fiber bundles. From now on, we apply the homogenization method to the governing equations of Stokes' flow to obtain the macroscopic equation and the microscopic equation of the mold flow. The macroscopic equation will be identical to Darcy's law except for the added sink term which represents the flux absorbed into the fiber bundle.

In order to apply the homogenization method, we expand the pressure and velocity into a formal power series of $\varepsilon$ (superscript $\varepsilon$ is added to $P$ and $v$ in order to represent the dependency in the microsopic level, $\bar{y}$ ):

$P^{\varepsilon}(\bar{x})=P^{(0)}(\bar{x})+\varepsilon P^{(1)}(\bar{x}, \bar{y})+\varepsilon^{2} P^{(2)}(\bar{x}, \bar{y})+\cdots$

$\bar{v}^{\varepsilon}(\bar{x})=\varepsilon^{2} \bar{v}^{(0)}(\bar{x}, \bar{y})+\varepsilon^{3} \bar{v}^{(1)}(\bar{x}, \bar{y})+\varepsilon^{4} \bar{v}^{(2)}(\bar{x}, \bar{y})+\cdots$

Fig. 6. Double porous fiber mat 
Here, $P^{(i)}$ and $\bar{v}^{(i)}$ are periodic functions with respect to argument $\bar{y}$. Introducing the asymptotic expansion (48)-(49) to

Eqs. (2)-(3) and collecting terms of the same order of $\varepsilon$ yields:

$-\frac{\partial P^{(1)}}{\partial y_{i}}+\frac{\partial}{\partial y_{j}}\left(\mu \frac{\partial v_{i}^{(0)}}{\partial y_{j}}\right)+\left(f_{i}-\frac{\partial P^{(0)}}{\partial x_{i}}\right)=0$

$\frac{\partial v_{i}^{(0)}}{\partial y_{i}}=0$

$\left.\bar{v}^{(0)}\right|_{\Gamma}=0$

$\frac{\partial v_{i}^{(0)}}{\partial x_{i}}+\frac{\partial v_{i}^{(1)}}{\partial y_{i}}=0$

$\left.\varepsilon^{3} v_{i}^{(1)} n_{i}\right|_{\Gamma}=\left.v_{i}^{d} n_{i}\right|_{\Gamma}$

Equations (50) - (54) are identical to Eqs. (10) - (14) in sect. 2.2.1 except for the boundary condition (54) which represents the mass balance between the two regimes of the flow domain.

The macroscopic and microscopic equations can be obtained by applying to Eqs. (50)-(54) the same procedure used in 2.2.1. They are:

- microscopic equation:

$\chi_{i}^{m} \in V_{y}: \quad \int_{Y_{1}} \frac{\partial \chi_{i}^{m}(\bar{y})}{\partial y_{j}} \frac{\partial w_{i}(\bar{y})}{\partial y_{j}} d Y=\int_{Y_{1}} w_{m}(\bar{y}) d Y \quad \forall w_{i} \in V_{y}$

where

$V_{y}=\left\{w_{i} \mid w_{i} \in\left(H^{1}\left(Y_{1}\right)\right)^{3}, Y\right.$-periodic, $w_{i}=0$ on $\left.\Gamma, \frac{\partial w_{i}}{\partial y_{i}}=0\right\}$

- macroscopic equation:

$\frac{\partial v_{i}^{D}}{\partial x_{i}}+\frac{\varepsilon^{2}}{|Y|} \int_{\Gamma_{Y}} v_{i}^{(1)} n_{i} d \Gamma=0$

where

$v_{i}^{D}=\frac{K_{i j}}{\mu}\left(f_{j}-\frac{\partial P}{\partial x_{j}}\right)$

$K_{i j}=\frac{\varepsilon^{2}}{|Y|} \int_{Y_{1}} \chi_{j}^{i} d Y$

$P=\frac{1}{|Y|} \int_{Y_{1}} P^{(0)} d Y$

$\left.P\right|_{Y_{1}}=\left.P\right|_{Y_{2}} \quad$ along $\Gamma$

$\int_{\Gamma} v_{i}^{(1)} n_{i} d \Gamma=-\left(\frac{\delta}{\varepsilon}\right)^{2} \int_{\Gamma} \frac{k}{\mu} \frac{\partial P}{\partial y_{i}} n_{i} d \Gamma$

The microscopic Eq. (55) can be solved for $\chi_{j}^{i}$, which in turn is used to calculate the permeability tensor $\left(K_{i j}\right)$ in Eq. (59). Using the permeability tensor, the macroscopic Eq. (57) is solved for the pressure distribution $(P)$ and the Darcean velocity $\left(v_{i}^{D}\right)$ of the mold flow. In addition, the actual flow field within the micro structure can be obtained by combining the solutions of both the macroscopic and microscopic equations:

$v_{i}^{\varepsilon} \approx \varepsilon^{2} v_{i}^{(0)}(\bar{x}, \bar{y})=\varepsilon^{2} \frac{\chi_{i}^{j}}{\mu}\left(f_{j}-\frac{\partial P}{\partial x_{j}}\right)$

While solving the macroscopic Eq. (57), it should be noted that the equation is coupled with Eq. (47) which is the governing equation of the resin flow within the fiber bundle. The coupling term, which is the second term of (57), represents the flux absorbed into the fiber bundle and must be evaluated from the solution of Eq. (47). To solve the coupled equations, one has to keep track of the moving free boundary within the fiber bundle while keeping track of the moving free boundary of the overall mold flow. This requires that if finite element approximation is to be used to solve (57), Eq. (47) must be solved at each node of the finite elements.

To indicate the degree of impregnation within the fiber bundle, a wet ratio can be introduced. The wet ratio, $\omega$ is defined as:

$\omega=\frac{\text { impregnated volume within the fiber bundle }}{\text { total volume of the fiber bundle }}$

Since the resin starts to impregnate the fiber bundle as soon as the fiber bundle is included in the flow domain, a wet ratio can also be used to determine the impregnated region within the mold cavity. The region can be identified by checking if the wet ratio is larger than zero at each point of the mold.

\section{2}

\section{The energy equation}

To obtain the averaged energy equation for a double porous fiber preform, the homogenization method should be applied twice, once in each regime of the flow domain. First, the method is applied to the energy equation within the fiber bundle which is the second level micro structure. As a result, the homogenized thermodynamic properties are obtained. They are:

$\left(\rho c_{p}\right)^{h}=\frac{1}{|Z|} \int_{Z} \rho c_{p} d Z$

$\dot{s}^{h}=\frac{1}{|Z|} \int_{Z} \dot{s} d Z$

$\kappa_{i p}^{h}=\frac{1}{|Z|} \int_{Z}\left(\kappa_{i p}+\kappa_{i j} \frac{\partial \psi^{p}}{\partial z_{j}}\right) d Z$

where $\psi^{p}$ is a solution of:

$\psi^{p} \in W_{z}: \quad \int_{Z} \frac{\partial \tau}{\partial z_{j}} \kappa_{i j} \frac{\partial \psi^{p}}{\partial z_{j}} d Z=-\int_{Z} \frac{\partial \tau}{\partial z_{i}} \kappa_{i p} d Z, \quad \forall \tau \in W_{z}$

$W_{z}=\left\{w_{i} \mid w_{i} \in\left(H^{1}(Z)\right)^{3}, Z\right.$-periodic $\}$

The formal derivations are identical to those of sect. 2.2.2 except for the scaling parameter $(\varepsilon)$ and thus omitted. The homogenized thermodynamic properties (65)-(67) represent the averaged thermodynamic properties of the impregnated 
part within the fiber bundle. $\left(\rho c_{p}\right)^{h}$ represent the averaged heat capacity, $\dot{s}^{h}$ represents the averaged heat source, and $\kappa_{i p}^{h}$ represents the averaged (or effective) heat conductivity.

Using these homogenized properties, the homogenization method is applied once again to the first level micro structure. This procedure is straightforward except for the region near the flow front where the fiber bundles are not fully impregnated. At this region, the local energy balance is made between the resin and the impregnated part of the fiber bundle. After applying the homogenization method once again to the energy equation, one obtains:

- microscopic equation:

$$
\begin{gathered}
\psi^{p} \in W_{y}: \int_{Y_{1}} \frac{\partial \tau}{\partial y_{i}} \kappa_{i j} \frac{\partial \psi^{p}}{\partial y_{j}} d Y+\int_{Y_{2 \omega}} \frac{\partial \tau}{\partial y_{i}} \kappa_{i j}^{h} \frac{\partial \psi^{p}}{\partial y_{j}} d Y \\
=-\int_{Y_{1}} \frac{\partial \tau}{\partial y_{i}} \kappa_{i p} d Y-\int_{Y_{2 \omega}} \frac{\partial \tau}{\partial y_{i}} \kappa_{i p}^{h} d Y, \quad \forall \tau \in W_{y} \\
W_{y}=\left\{w_{i} \mid w_{i} \in\left(H^{1}(Y)\right)^{3}, Y \text {-periodic }\right\}
\end{gathered}
$$

- macroscopic equation:

$$
\begin{gathered}
\int_{\Omega}\left(\rho c_{p}\right)^{H} \frac{\partial T^{(0)}}{\partial t} \tau d \Omega+\int_{\Omega} \rho_{r} c_{p r} \nu_{i}^{D} \frac{\partial T^{(0)}}{\partial x_{i}} \tau d \Omega+\int_{\Omega} \kappa_{i p}^{H} \frac{\partial T^{(0)}}{\partial x_{i}} \frac{\partial \tau}{\partial x_{p}} d \Omega \\
=\int_{\Omega} \dot{s}^{H} \tau d \Omega-\int_{\Omega} \phi_{f}^{(1)} \phi_{f}^{(2)} \rho_{f} c_{p f} \frac{d \omega}{d t}\left(T^{(0)}-T_{g}\right) \tau d \Omega \\
\left(\rho c_{p}\right)^{H}=\frac{1}{|Y|}\left[\int_{Y_{1}} \rho c_{p} d Y+\int_{Y_{2 \omega}}\left(\rho c_{p}\right)^{h} d Y\right] \\
\kappa_{i p}^{H}=\frac{1}{|Y|}\left[\int_{Y_{1}}\left(\kappa_{i p}+\kappa_{i j} \frac{\partial \psi^{p}}{\partial y_{j}}\right) d Y+\int_{Y_{2 \omega}}\left(\kappa_{i p}^{h}+\kappa_{i j}^{h} \frac{\partial \psi^{p}}{\partial y_{j}}\right) d Y\right] \\
\dot{s}^{H}=\frac{1}{|Y|}\left[\int_{Y_{1}} \dot{s} d Y+\int_{Y_{2 \omega}} \dot{s}^{h} d Y\right]
\end{gathered}
$$

where $Y_{2 \omega}$ is the impregnated part of the fiber bundle $Y_{2}$. $\phi_{f}^{(1)}$ and $\phi_{f}^{(2)}$ are the volume fraction of the fiber part in the first and second levels of the micro structure. They are defined as $\left|Y_{2}\right| /|Y|$ and $\left|Z_{2}\right| /|Z|$ respectively.

The microscopic Eq. (70) has the same form as that of the porous fiber preform except for the integration domain. In (70), the integration domain is $Y_{1} \cup Y_{2 \omega}$, whereas in the porous fiber preform, it is $Y\left(Y=Y_{1} \cup Y_{2}\right)$. The macroscopic Eq. (72) has the same from as that of the porous fiber preform except for the last term which takes into account the initial energy of the fiber preform. In the double porous flow domain, the local energy balance is made considering the degree of impregnation within the fiber bundle. The homogenized thermodynamic properties (73)-(75) also have the same form as those of the porous fiber preform except for the integration domain which is $Y_{1} \cup Y_{2 \omega}$ instead of $Y_{1} \cup Y_{2}$.

\section{4}

\section{Numerical implementation}

The numerical implementations of microscopic Eqs. (55), (68), and (70), which have the same form, can be easily achieved using the conventional finite element method. In contrast, the numerical implementation of macroscopic Eqs. (57) and (72) poses several difficulties. Most of the difficulties come from the fact that the mold filling is a transient process throughout which the flow domain is changing constantly. To solve the macroscopic equations, one has to find the location of the flow front and redefine the meshes at each time step. In the finite element method, this requires iterations to keep the flow front within the mold cavity and a re-meshing at each time step, which significantly slow down the simulation. See Lee and $\operatorname{Sin}(1990)$.

In order to avoid the re-meshing at each time step, several numerical methods have been developed. Examples of these methods are the Fluid Analysis Network (FAN) method (Tadmor, Broyer and Gutfinger (1974)) and the Finite Element/Control Volume (FE/CV) method (Wang, Hieber and Wang (1986)). In these methods, One initial grid is used throughout the entire simulation, and the flow front can be found without any iteration. In the following sections, the numerical implementations of both macroscopic Eqs. (57) and (72) are discussed using the $\mathrm{FE} / \mathrm{CV}$ method.

4.1 The homogenized momentum and continuity equations Macroscopic Eq. (57) is coupled with Eq. (47) which governs the flow within the fiber bundle. In order to solve Eq. (57) using finite element approximation, one first has to solve the coupled Eq. (47) and keep track of the moving free boundary within the fiber bundle at each nodal point of the finite elements. This can be done by updating the location of the flow front at each time step according to the time increment and the Darcean velocity obtained from the solution of Eq. (47). Then, using this solution, one has to solve Eq. (57) over the entire flow domain which is changing constantly throughout the mold filling process.

Here, an analytical solution of (47) is found and utilized while the $\mathrm{FE} / \mathrm{CV}$ method is utilized to solve (57). An analytical solution can be found when the fiber bundle has a rather simple geometry. For a fiber bundle having a circular cross section as shown in Fig. 8, the pressure is found to have logarithmic distribution:

$P_{Y_{2}}=P\left(-\frac{1}{\ln \left(r_{i} / r_{o}\right)} \ln \left(\frac{r}{r_{0}}\right)+1\right)$

where $P_{Y_{2}}$ is the pressure within the fiber bundle and $P$ is the

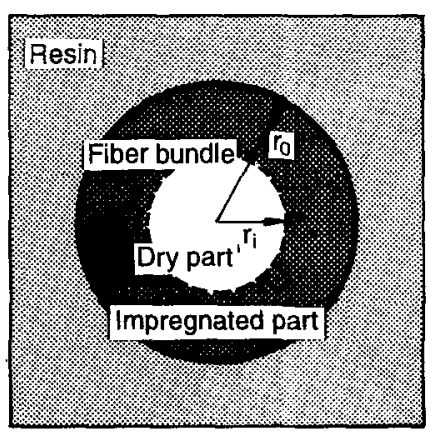

Fig. 8. Cross section of a circular fiber bundle 
resin pressure surrounding the fiber bundle. $r_{i}$ represents the location of the flow front, and $r_{o}$ represents the radius of the fiber bundle in stretched coordinates. For a more complicated geometry, a numerical solution should be found using the FE/CV method.

FE/CV method can be used to solve the macroscopic Eq. (57). In the FE/CV method, the mold cavity is first divided into eight node hexagonal elements. The centroids of each face of these elements are joined to the midpoints of the edges and to the centroid of the element, creating polygonal control that surround each vertex node. This is shown in Fig. 9.

The FE/CV method is based on the "conservation of mass principle" applied to each control volume. Equation (57), can be interpreted as: "the net flux entering the control volume must be equal to the amount of flux absorbed into the fiber bundles within the control volume". This interpretation is used to construct a set of linear equations which is the discretized version of Eq. (57).

The net flux entering each control volume can be obtained by integrating the flux over the boundary of the control volume. As shown in Fig. 10, the control volume assigned to a node is accommodated by several elements surrounding that node. Within each element, pressure distribution is interpolated using tri-linear shape functions. From the pressure distribution, the flow rate can be obtained which is proportional to the gradient of the pressure distribution. By integrating the flow rate over the boundaries of the control volume, the net flow rate into the control volume can be obtained in terms of nodal pressures. The net flow rate entering the control volume can be represented as:

$q=\sum_{i=1}^{n e l} \int_{A i}\left[-K_{m n}\left(f_{n}-\frac{\partial N_{l}}{\partial x_{n}} P_{l}\right) \hat{n}_{m}\right] d A$

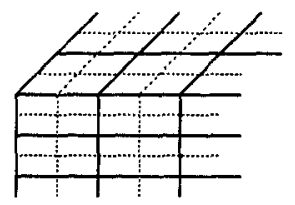

- Element boundary ........ Control volume boundary

Fig. 9. Finite elements and control volumes

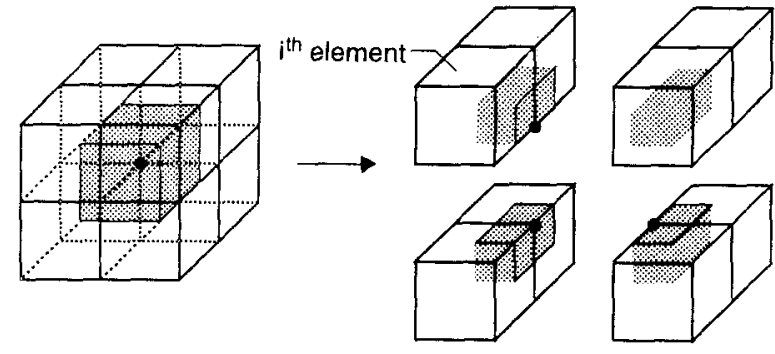

Fig. 10. Control volume assigned to a node where $P_{l}$ is the nodal pressure, $N_{l}$ is the tri-linear shape function, and $\hat{n}_{m}$ is the outward normal vector. nel is the total number of elements accommodating the control volume and $A_{i}$ is the boundary of the control volume contained in the $i$ th element.

The amount of flux absorbed into the fiber bundles within each control volume can be obtained by integrating the second term of Eq. (57) over the control volume. This can be done by integrating the term within each element using the tri-linear pressure distribution and summing up over the elements containing the control volume.

The boundaries of the flow domain include the mold walls, the injection ports, and the flow front. At the mold walls, the normal component of the flux is set to zero so that the flux is always parallel to the walls. At the injection ports, either pressure is specified or incoming flux is assigned according to the type of injection ports. At the flow front, a parameter $\vartheta$ is used to represent the status of each control volume. If a control volume is empty, $\vartheta$ is set to zero. If a control volume is filled up with resin, $\vartheta$ is set to one. If a control volume is partially filled, $\vartheta$ is set to the volume fraction of the resin within the control volume. The control volumes with $\vartheta$ between one and zero are considered to be at the flow front, and zero pressure is assigned to the corresponding node.

A set of algebraic equations can be constructed by considering the mass conservation principle with the boundary conditions above. Since both the net flux entering a control volume and the net flux absorbed into the fiber bundles within the control volume can be represented in terms of nodal pressures $P_{l}$, the resulting equations are in terms of nodal pressures. By solving the set of linear equations, the pressure distribution can be obtained. The pressure distribution is then used to determine the velocity of the moving flow front which is needed to determine the location of the moving flow front at the next time step.

The mold filling process can be regarded as a quasi-steady state process which assumes a steady condition at each time step. With the velocity of the flow front and the time increment, the location of the flow front can be updated at each time step. The selection of time increment for each quasi-steady state is based on two considerations. One is that each time increment allow less than one control volume to be completely filled and the other is that each time increment be small enough to ensure the accuracy of the solution within the fiber bundles. These restrictions on the time increment ensure the stability of the quasi-steady state approximation.

\section{2}

\section{The homogenized energy equation}

Macroscopic Eq. (72) of the energy equation can be discretized using the FE/CV method with the Crank-Nicholson method, which is one of the time integration methods. This approach considers the energy balance for each control volume to construct the discretized version of (72). The approach is not only consistent with the previous section (sect. 4.1 ), but also has the advantage that it is unconditionally stable and has small upwinding effects.

Since each control volume is assigned to a node lying at the center of the control volume, the energy balance for each control volume yields a linear equation in terms of the nodal temperature at the center of each control volume. The energy 
balance for a control volume $i$ is:

$$
\begin{aligned}
c_{1}\left(V^{n+1} T_{i}^{n+1}-V^{n} T_{i}^{n}\right)+c_{2} V^{n}\left(\omega^{n+1} T_{i}^{n+1}-\omega^{n} T_{i}^{n}\right) \\
\quad-c_{3} V^{n}\left(\omega^{n+1}-\omega^{n}\right) T_{g} \\
=\sum_{j=1}^{N} \int_{A_{j}}-T^{n+0.5} \rho_{r} c_{p r} v_{i}^{D} \hat{n}_{i} d A \Delta t \\
\quad+\sum_{j=1}^{N} \int_{A_{j}}-\kappa_{m n}^{H}\left(f_{n}-\frac{\partial T^{n+0.5}}{\partial x_{n}}\right) \hat{n}_{m} d A \Delta t \\
\quad+\left(c_{1}+c_{2}\right) \frac{\left(V^{n+1}+V^{n}\right)}{2} \dot{s} \Delta t
\end{aligned}
$$

where the superscript $n$ denotes the values at the current time step and $(n+1)$ denotes the values at the next time step. $V$ denotes the fraction of the control volume filled by the resin and $T_{i}$ is the nodal temperature. $\omega$ represents the wet ratio as defined in (64). $T_{g}$ is the initial temperature of the fiber preform. $c_{1}, c_{2}$ and $c_{3}$ are the thermal properties of the fiber and resin. They are defined as:

$c_{1}=\phi_{r}^{(1)} \rho_{r} c_{p r}$

$c_{2}=\left(1-\phi_{r}^{(1)}\right) \phi_{r}^{(2)} \rho_{r} c_{p r}$

$c_{3}=\left(1-\phi_{r}^{(1)}\right)\left(1-\phi_{r}^{(2)}\right) \rho_{f} c_{p f}$

where $\phi_{r}^{(1)}$ and $\phi_{r}^{(2)}$ represent the porosity (or volume fraction of the resin part) in the first level micro structure and in the second level micro structure respectively. $\hat{n}_{i}$ is the outward normal vector and $\Delta t$ is the time step. The convection and conduction terms are taken into account by integrating the incoming heat flux over the surface areas $A_{j}$ which are contained in each $N$ surrounding elements. The variables are evaluated at surface $A_{j}$ using tri-linear shape functions and integrated using the Gaussian integration. In (78), $T^{n+0.5}$ and $\partial T^{n+0.5} / \partial x_{m}$ are given as:

$T^{n+0.5}=\left.\frac{1}{2}\left(T^{n}+T^{n+1}\right)\right|_{A i}$

$\frac{\partial T^{n+0.5}}{\partial x_{m}}=\left.\frac{1}{2}\left[\left(\frac{\partial T}{\partial x_{m}}\right)^{n}+\left(\frac{\partial T}{\partial x_{m}}\right)^{n+1}\right]\right|_{A_{i}}$

A set of linear equations can be obtained in terms of nodal temperatures by applying the energy balance (78) to each control volume. By solving the set of equations, the temperature distribution can be obtained which is used to determine the viscosity of the resin.

\section{3}

\section{Solution procedure}

The flow chart for the numerical simulation program is shown in Fig. 11. The input data include: the mesh data, material properties of the fiber preform and resin, injection parameters, and initial conditions. With this information, the viscosity is calculated which is used to find the pressure distribution. The pressure field, in turn, is used to calculate the flow front velocities in the first level micro structure and in the second

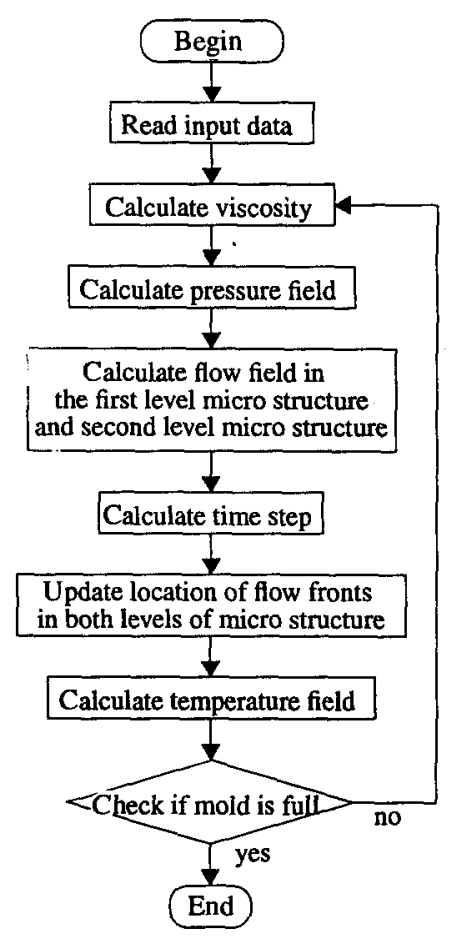

Fig. 11. Flow chart of the mold filling simulation program

level microstructure. With the flow front velocities, the time step is determined and the flow domains are updated in both levels of micro structure. In the updated flow domain, the temperature field is calculated. Then the program checks if the mold is completely full. If the mold is not full, the program calculates the resin viscosity and repeats the cycle until the mold is completely full.

It should be noted that even though the energy equation is coupled with the momentum and continuity equations, iteration is not used to solve these coupled equations. The heat transfer (or the temperature field) affects the flow field through viscosity and the flow field, in turn, affects the heat transfer through convection of the resin. An iteration algorithm can be used to solve the coupled equations. However, in the current analysis this is not necessary because it is time consuming and does not significantly enhance the accuracy of the solution.

\section{5}

\section{Example}

A mold filling process is simulated for a composite crossmember of a passenger van (Farris (1987)). Due to the complexity of the geometry, a simplified model is used in the simulation. The corresponding mold cavity is shown in Fig. 12. The mold cavity has a shell type geometry surrounding the foam core. It is packed with a double porous fiber preform whose micro structure is shown in Fig. 13. At the center of the front mold wall is the injection port through which the cold resin $\left(25^{\circ} \mathrm{C}\right)$ is injected at $0.5 \times 10^{-4} \mathrm{~m}^{3} / \mathrm{s}$. The mold is assumed to be heated so that the initial temperature of the fiber preform is $140^{\circ} \mathrm{C}$ and the temperature of the mold walls is fixed at $140^{\circ} \mathrm{C}$ throughout the process. In the actual process, however, the temperature at the mold walls may vary. In a case where the temperature variation is significant, the mold plates should 


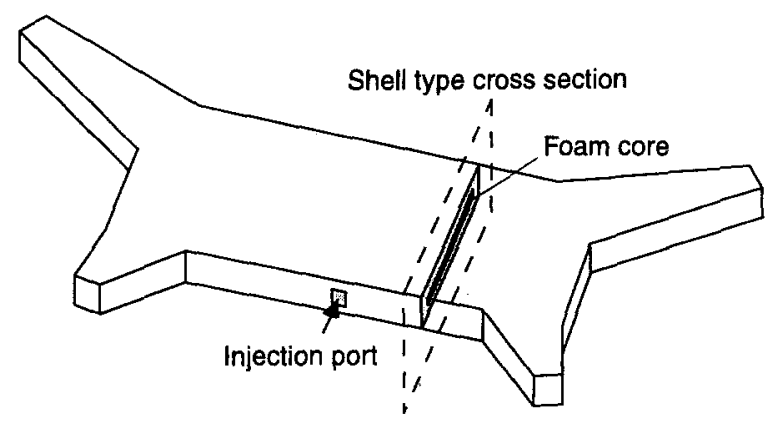

Fig. 12. Configuration of the mold cavity for a simplified crossmember

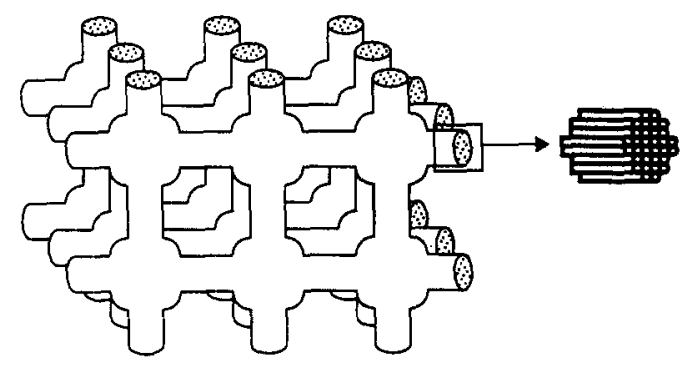

Fig. 13. Micro structure of the double porous fiber preform used in the example

be included in the heat transfer analysis. The parameters used in the simulation are:

- volume fraction of the resin part:

$\phi_{r}^{(1)}=0.7$

$\phi_{r}^{(2)}=0.4$

- resin properties

$\rho_{r}=1140\left(\mathrm{Kg} / \mathrm{m}^{3}\right)$

$c_{p r}=1900\left(\mathrm{~J} /\left(\mathrm{Kg}^{\circ} \mathrm{K}\right)\right)$

$\kappa_{r}=0.2\left(\mathrm{~W} /\left(\mathrm{m}^{\circ} \mathrm{K}\right)\right)$

$\mu= \begin{cases}0.1 \exp (-0.048(T-32))(P a \cdot s), & \left(T \leqq 80^{\circ} \mathrm{C}\right) \\ 0.00998 \exp (-0.0115(T-80))(P a \cdot s), & \left(T>80^{\circ} \mathrm{C}\right)\end{cases}$

- fiber properties

$\rho_{f}=2500\left(\mathrm{Kg} / \mathrm{m}^{3}\right)$

$c_{p r}=900\left(\mathrm{~J} /\left(\mathrm{Kg}^{\circ} \mathrm{K}\right)\right)$

$\kappa_{f}=1.0\left(\mathrm{~W} /\left(\mathrm{m}^{\circ} \mathrm{K}\right)\right)$

The permeability and the conductivity within the mold cavity are calculated by solving the corresponding microscopic equations. The finite element meshes used in the calculation is shown in Figs. 14 and 15. The permeabilities and the conductivity are:

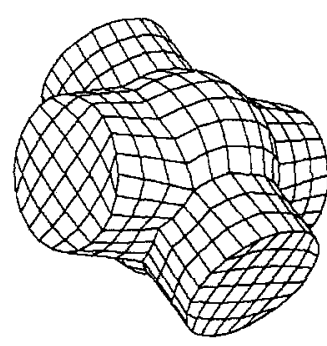

Fiber bundle part

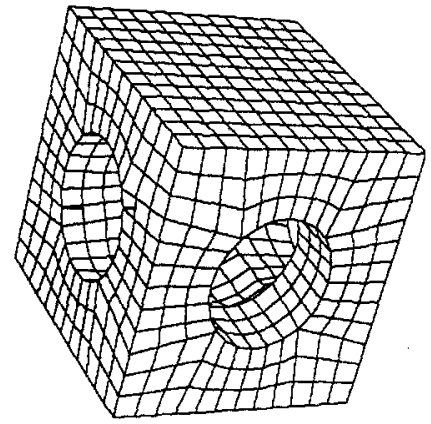

Resin part
Fig. 14. Finite element meshes of the first level micro structure

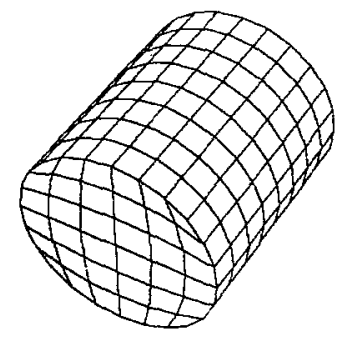

Fiber part

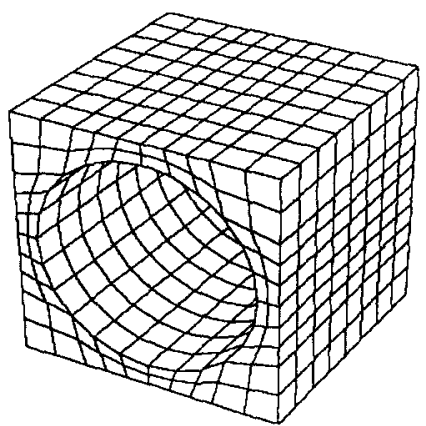

Resin part
Fig. 15. Finite element meshes of the second level micro structure

- calculated permeabilities:

$K=\left[\begin{array}{lll}0.188 & 0.000 & 0.000 \\ 0.000 & 0.188 & 0.000 \\ 0.000 & 0.000 & 0.720\end{array}\right] \times 10^{-6}\left(\mathrm{~m}^{2}\right)$

$k=0.100 \times 10^{-2}\left(\mathrm{~m}^{2}\right)^{1}, \quad \varepsilon=0.004 \quad \delta=0.025$

- calculated homogenized conductivity:

$\kappa=\left[\begin{array}{lll}0.313 & 0.000 & 0.000 \\ 0.000 & 0.313 & 0.000 \\ 0.000 & 0.000 & 0.256\end{array}\right]\left(\mathrm{W} /\left(\mathrm{m}^{\circ} \mathrm{K}\right)\right)$

The homogenized conductivity $(\kappa)$ is calculated assuming that the inside of the reinforcing fiber bundle is fully impregnated. At the region near the flow front where the inside of the reinforcing fiber bundle is not fully impregnated (wet ratio $<1$ ), the conductivity depends on the degree of impregnation. In the current example, this effect is neglected because the region is small and does not significantly affect the overall heat transfer. In the simulation, only quarter part of the mold is used because the mold cavity has a symmetric geometry. The finite element meshes used are shown in Fig. 16.

It should be noted that, in the simulation, many simplifications are made due to the lack of experimental data

\footnotetext{
'see equation (47)
} 


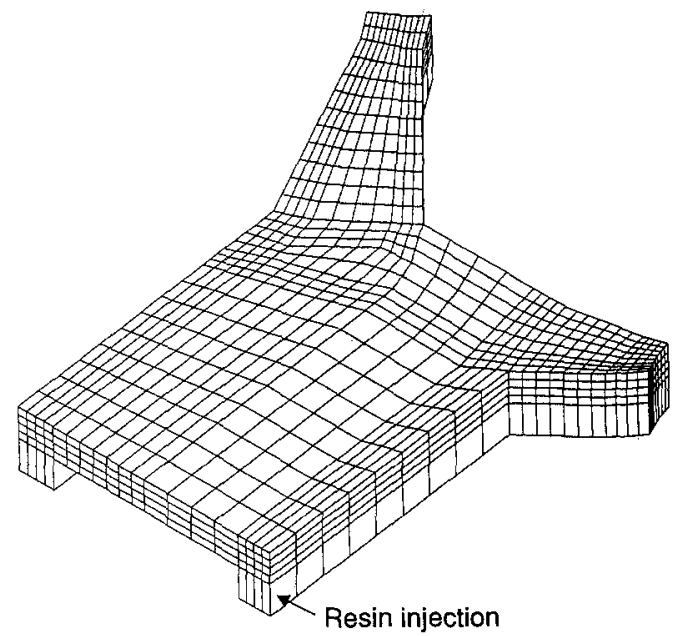

Fig. 16. Finite element meshes of the quarter part of the mold cavity

and/or for the sake of simplicity: the effect of surface tension for the flow inside the fiber bundles is neglected; change of viscosity due to the partial cure of resin is neglected; and the mold wall temperatures are set to constant. These effects, however, can be added without much difficulty for more accurate analysis. The current example is intended to serve as to demonstrate the strength of the analysis method rather than to give a complete analysis.

In the simulation, the flow front profile, temperature distribution, and wet ratio are calculated at various points of the mold filling. In addition, actual flow field within the micro structure is calculated using the solutions of the microscopic equations. Figure 17 shows the flow front profiles within the mold cavity. The boundaries of each shaded region represents the flow fronts when the mold is partially filled, and the numbers represent the volume fraction (fill ratio) of the impregnated region. Figure 18 shows the temperature distribution which is obtained by plotting the temperatures at the center of each element when the mold is $50 \%$ full. The distribution shows that the temperature is higher near the flow front than near the injection port and higher at the mold walls than at the center

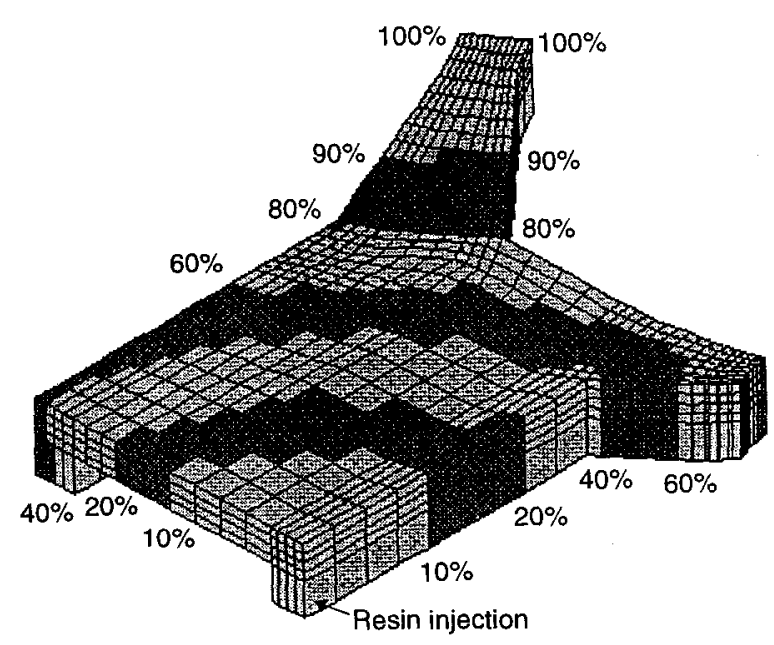

Fig. 17. Flow fronts at various fill ratio
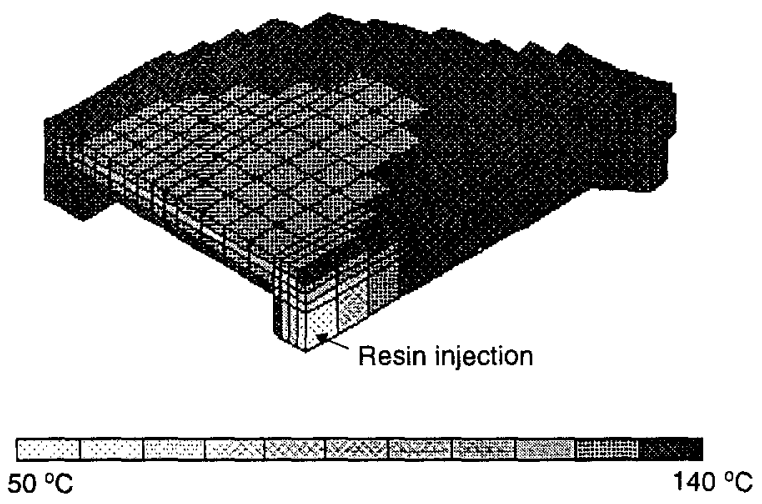

Fig. 18. Temperature distribution when the mold is $50 \%$ full

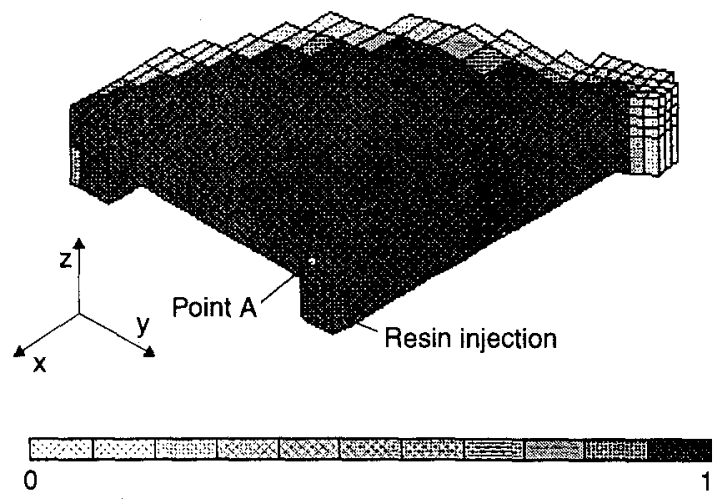

Fig. 19. Wet ratio when the mold is $50 \%$ full

of mold cavity, which is typical when cold resin is injected into a heated mold. Figure 19 shows the wet ratio of the impregnated region when the mold is $50 \%$ full. The wet ratio is less than one at the flow fronts, which implies that the inside of the reinforcing fiber bundle is not fully impregnated.

Figure 20 shows the actual flow field within the micro structure at point $\mathrm{A}$ (shown in Fig. 19) when the mold is $50 \%$ full. The arrows show the velocity of resin moving around the reinforcing fiber bundle.

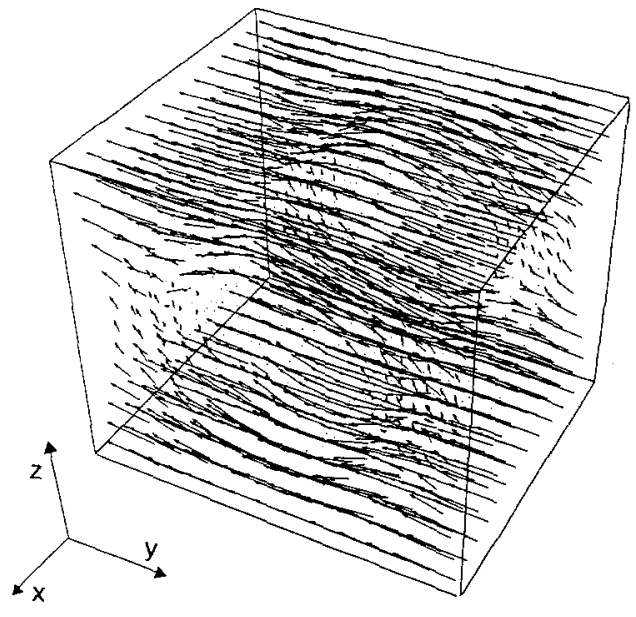

Fig. 20. Actual flow field within the micro structure point $A$ when mold is $50 \%$ full 


\section{Conclusion}

Using the homogenization method, the macroscopic and microscopic equations are derived for the non-isothermal mold filling process with double porous fiber preforms. The macroscopic equations governs the averaged heat and mass transport during the mold filling process, whereas the microscopic equations governs the characteristic behavior within the micro structure. The microscopic equations are solved using the finite element method, and their solutions are used to calculate the permeabilities and the averaged (or effective) heat conductivity which, in tern, are used to solve the macroscopic equations in the simulation program. A 3-D simulation program based on the finite element/control volume method and the Crank Nicholson method is developed which is capable of predicting the flow pattern and the temperature distribution during the mold filling process. By post-processing the solutions of the microscopic equations and the macroscopic equations, the actual flow field within the micro structure is obtained.

\section{References}

Arbogast, T. 1989: Analysis of the simulation of single phase flow through a naturally fractured reservoir, SIAM J. Numer. Anal. 26: 12-29 Bruschke, M. V.; Advani, S. G. 1990: A finite element/control volume approach to mold filling in anisotropic porous media, Polymer Composites 11: 398-405

Bruschke, M. V.; Advani, S. G. 1993: A numerical approach to model non-isothermal viscous flow through fibrous media with free surfaces, International Journal for Numerical Methods in Fluids, to appear Chan, A. W.; Hwang, S.-T. 1988: Mold filling simulations for the injection molding of continuous fiber-reinforced polymer, Polymer Eng. and Sci. 26: 333-339

Coulter, J. P.; Güçeri, S. I. 1988: Resin impregnation during the manufacturing of composite materials subject to prescribed injection rate, Journal of Reinforced Plastics and Composites 7: 200-219

Davé, R. 1990: A unified approach to modeling resin flow during composite processing, Journal of composite materials 24: 22-41

Farris, R. D. 1987: Composite front crossmember for the Chrysler T-115 mini-van, Proceedings of the Third Annual Conference on Advanced composites, 15-17, Detroit, Michigan

Gonzalez, V. M.; Castro, J. M.; Macosko, C. W. 1985: Polymer Process Engineering 3: 173

Hieber, C. A.; Shen, S. F. 1978: Flow analysis of the non-isothermal two dimensional filling process in injection molding process, Israel journal of technology 16: 248-254

Hieber, C. A.; Shen, S. F. 1980: A finite element/finite difference simulation of the injection-molding filling process, J. of Non-

Newtonian Fluid Mech. 7: 1-32

Hornung, U. 1991: Homogenization of miscible displacement in unsaturated aggregated soils. In: Gianni Dal Maso and Gian Fausto Dell Antonio (ed): Composite Media and Homogenization Theory, 143-152, Boston: Birkhäuser

Kaviany, M. 1991: Principles of Heat Transfer in Porous Media, 26-42, New York: Springer-Verlag

Keller, J. B. 1980: Darcy's law for flow in porous media and the twospace method. In: Sternberg, R. L.; Kalinowski, A. J.; Papadakis, J. S. (ed): Nonlinear partial differential equations in engineering and applied science, 429-443, New York: M. Dekker

Lee, C. 1990: An improved Flow Analysis Network (FAN) for irregular geometries, Polymer Engineering and Science 30: 1607-1614

Lee, H.; Sin, H. 1990: Iterative boundary pressure reflection method for the simulation of injection mold filling, Polymer Engineering and Science 30: 1513-1522

Lene, F. 1984: Thése de Doctorat d'Etat, Université Pierre et Marie Curie, Paris VI

Lions, J. L. 1981: Some methods in the mathematical analysis of systems and their control, New York: Science Press

Tadmor, Z.; Broyer, E.; Gutfinger, C. 1974: Flow Analysis Network (FAN)-A method for solving flow problems in polymer processing, Polymer Engineering and Science 14: 660-665

Tartar, L. 1980: Incompressible fluid flow in a porous mediumconvergence of the homogenization process. In: Enrique SanchezPalencia (ed.): Non homogeneous media and vibration theory, 368-377, Berlin: Springer Verlag

Trochu, F.; Gauvin, R. 1992: Some issues about the numerical simulation of mold filling in resin transfer molding, Advanced composite letters 1: 41-43

Wang, V. W.; Hieber, C. A.; Wang, K. K. 1986: Dynamic simulation and graphics for the injection molding of three-dimensional thin parts, J. of Polymer Eng. 7: 21-45

Winslow, A. M. 1967: Numerical solution of the quasi-linear Poisson equation in a nonuniform triangle mesh, Journal of computational physics 2: 149-192

Young, W. B.; Han, K.; Fong, L. H.; Lee, L. J. 1991: Flow simulation in molds with preplaced fiber mats, Polymer composites 12: $391-403$ 